\title{
Constraints on the speed of sound of dense nuclear matter through the tidal deformability of neutron stars
}

\author{
Alkiviadis Kanakis-Pegios ${ }^{1, *}$, Polychronis Koliogiannis ${ }^{1}$, and Charalampos Moustakidis ${ }^{1}$ \\ ${ }^{1}$ Department of Theoretical Physics, Aristotle University of Thessaloniki, Thessaloniki, Greece
}

\begin{abstract}
One of the greatest interest and open problems in nuclear physics is the upper limit of the speed of sound in dense nuclear matter. Neutron stars, both in isolated and binary system cases, constitute a very promising natural laboratory for studying this kind of problem. This present work is based on one of our recent study, regarding the speed of sound and possible constraints that we can obtain from neutron stars. To be more specific, in the core of our study lies the examination of the speed of sound through the measured tidal deformability of a binary neutron star system (during the inspiral phase). The relation between the maximum neutron star mass scenario and the possible upper bound on the speed of sound is investigated. The approach that we used follows the contradiction between the recent observations of binary neutron star systems, in which the effective tidal deformability favors softer equations of state, while the high measured masses of isolated neutron stars favor stiffer equations of state. In our approach, we parametrized the stiffness of the equation of state by using the speed of sound. Moreover, we used the two recent observations of binary neutron star mergers from LIGO/VIRGO, so that we can impose robust constraints on the speed of sound. Furthermore, we postulate the kind of future measurements that could be helpful by imposing more stringent constraints on the equation of state.
\end{abstract}

\section{Introduction}

One of the current unsolved problems in Nuclear Physics is the properties of dense nuclear matter. Neutron stars are among the most promising sources in universe for studying the dense nuclear matter [1-4]. The speed of sound consists a microscopic quantity of great interest, especially the possible upper bounds that can get, since it affects the equation of state $(\mathrm{EoS})$.

The main assumption about the upper bound of the speed of sound is that fullfills the causality, i.e. the speed of sound cannot exceed the speed of light, which is the main restriction in the speed of sound imposed from general principles according to Zel'dovich [5, 6]. Various studies have been made about the upper bound on the speed of sound (see Refs. [4, 7, 8]). Furthermore, in Ref. [8] the authors found also that the existence of neutron stars with masses about two solar masses, combined with the knowledge of the EoS of hadronic matter at low densities, is not consistent with the bound $c / \sqrt{3}$. The speed of sound and its effects on the tidal deformability has been studied also in Refs. [9-12].

We notice that there are two main considerations for the possible upper bound in the speed of sound of dense nuclear matter. Firstly, the causality which imposes an upper bound on the speed of sound $v_{c}=c$, and secondly a conformal upper limit with $v_{c}=c / \sqrt{3}$. Additionally, the limit $v_{s}=c / \sqrt{3}$ is related to the existence of quark matter in neutron star interior, consisting a very important rea-

*e-mail: alkanaki@auth.gr son to study this case of speed of sound bound (for more details see Refs. [13, 14]).

The main idea that motivated our study is the examination of possible constraints on the tidal deformability of neutron stars through the speed of sound and its upper bounds. We notice that there is a contradiction that arises regarding the stifness of the EoS; the recent high measured values of neutron star masses favor stiff EoS, while the upper limit on the effective tidal deformability (from gravitational-wave detections) favors softer EoSs. Our study aims to shed more light on these antagonized points of view, by parametrizing the stifness (softness) of the EoS via the speed of sound upper bounds and the transition density. The predictions are combined with the recent observations of two events, GW170817 and GW190425 [1518 ], as well as the current observed maximum neutron star masses $\left(1.908 \pm 0.016 M_{\odot}[19], 2.01 \pm 0.04 M_{\odot}\right.$ [20], $2.14_{-0.09}^{+0.10} M_{\odot}$ [21], 2.27 ${ }_{-0.15}^{+0.17} M_{\odot}$ [22]) (see also Ref. [23]).

The demand of a) a soft EoS for low densities (in order to be in accordance with the upper limit of the tidal deformability) and b) a stiff EoS for high densities (in order to predict the high neutron star measurements) leads to robust constraints on the EoS. In addition, we postulate what kind of future measurements could help us to more stringent constraints. For more details, we refer to the original study Ref. [24].

Moreover, we mention the recent detection of GW190814 event [25, 26], in which the lighter component compact object might be a neutron star. Following 
this hypothesis, in a recent work we studied the role of the speed of sound in such a case [27].

\section{Theoretical background}

\subsection{Speed of sound and EoS}

First of all, we constructed the necessary maximum mass configuration, by parametrizing the neutron star EoS as follows [28-36]

$$
P(\mathcal{E})=\left\{\begin{array}{l}
P_{\text {crust }}(\mathcal{E}), \quad \mathcal{E} \leq \mathcal{E}_{\mathrm{c}-\text { edge }} \\
P_{\mathrm{NM}}(\mathcal{E}), \quad \mathcal{E}_{\mathrm{c}-\text { edge }} \leq \mathcal{E} \leq \mathcal{E}_{\mathrm{tr}} \\
\left(\frac{v_{\mathrm{s}}}{c}\right)^{2}\left(\mathcal{E}-\mathcal{E}_{\mathrm{tr}}\right)+P_{\mathrm{NM}}\left(\mathcal{E}_{\mathrm{tr}}\right), \quad \mathcal{E}_{\mathrm{tr}} \leq \mathcal{E} .
\end{array}\right.
$$

where $P$ and $\mathcal{E}$ are the pressure and energy density, respectively, and $\mathcal{E}_{\text {tr }}$ is the transition energy density. In region $\mathcal{E} \leq \mathcal{E}_{\text {c-edge }}$, we used the equation of Feynman et al. [37] and also of Baym et al. [38] for the crust and low densities of neutron star. In the intermediate region, $\mathcal{E}_{\text {c-edge }} \leq \mathcal{E} \leq \mathcal{E}_{\text {tr }}$, we employed a specific EoS based on the MDI model and data from Akmal et al. [39], while for $\mathcal{E}_{\text {tr }} \leq \mathcal{E}$, the EoS is maximally stiff with the speed of sound, defined as $v_{s}=c \sqrt{(\partial P / \partial \mathcal{E})_{S}}$ (where $S$ is the entropy). In the framework of the present work, the speed of sound is fixed on the two values, $c / \sqrt{3}$ and $c$. The cases which took effect in this study were the ones where the fiducial baryon transition density is $n_{\mathrm{tr}}=p n_{0}$, where $n_{0}$ is the saturation density of symmetric nuclear matter $\left(n_{0}=0.16 \mathrm{fm}^{-3}\right)$ and $p$ takes the values $1,1.5,2,3$. Therefore, the predicted EoSs are functional of $n_{\mathrm{tr}}$ and $v_{s}$ and implemented to study their effects on the bulk neutron star properties including $M_{\max }$ (maximum mass of a neutron star), and $\Lambda$ (tidal deformability; for definition see below Eq. (10)).

While in approach (1) the continuity on the EoS is well ensured, its artificial character does not ensure the continuity in the speed of sound at the transition density. We underline that the speed of sound must be treated carefully, especially in regions of discontinuity [40]. Therefore, in order to ensure the continuity and a smooth phase transition, a method is needed, in which we considered that above the transition density, the speed of sound is parametrized as follows (for more details see Ref. [41])

$$
\frac{v_{\mathrm{s}}}{c}=\left(a-c_{1} \exp \left[-\frac{\left(n-c_{2}\right)^{2}}{w^{2}}\right]\right)^{1 / 2}, \quad a=1,1 / 3
$$

where the parameters $c_{1}, c_{2}$, and $w$ are fit to the speed of sound and its derivative at $n_{\text {tr }}$, and also to the demands $v_{\mathrm{S}}\left(n_{\mathrm{tr}}\right)=[c, c / \sqrt{3}]$ [28]. Using Eq. (2), the EoS for $n \geq n_{\text {tr }}$ can be constructed with the help of the following recipe [41]

$$
\begin{gathered}
\mathcal{E}_{i+1}=\mathcal{E}_{i}+\Delta \mathcal{E}, \quad P_{i+1}=P_{i}+\left(\frac{v_{s}}{c}\left(n_{i}\right)\right)^{2} \Delta \mathcal{E}, \\
\Delta \mathcal{E}=\Delta n\left(\frac{\mathcal{E}_{i}+P_{i}}{n_{i}}\right), \\
\Delta n=n_{i+1}-n_{i} .
\end{gathered}
$$

\subsection{Tidal deformability of neutron stars}

One of the most important sources for ground-based gravitational wave detectors are the gravitational waves from the final stages of inspiraling binary neutron stars [40, 4249]. Especially, Flanagan and Hinderer [43] articulated that tidal effects are also potentially measurable during the early part of the evolution when the waveform is relatively clean. The response of a component neutron star in a binary neutron star system to the tidal field is described by the tidal Love number $k_{2}$, which relates the quadrupole moment $Q_{i j}$ to the applied tidal field $E_{i j}[43,50]$

$$
Q_{i j}=-\frac{2}{3} k_{2} \frac{R^{5}}{G} E_{i j} \equiv-\lambda E_{i j}
$$

where $R$ is the neutron star radius and $\lambda=2 R^{5} k_{2} / 3 G$ is the tidal deformability. The tidal Love number $k_{2}$ is given by $[43,44]$

$$
\begin{aligned}
k_{2} & =\frac{8 \beta^{5}}{5}(1-2 \beta)^{2}\left[2-y_{R}+\left(y_{R}-1\right) 2 \beta\right] \\
& \times\left[2 \beta\left(6-3 y_{R}+3 \beta\left(5 y_{R}-8\right)\right)\right. \\
& +4 \beta^{3}\left(13-11 y_{R}+\beta\left(3 y_{R}-2\right)+2 \beta^{2}\left(1+y_{R}\right)\right) \\
& +3(1-2 \beta)^{2} \\
& \left.+\left[2-y_{R}+2 \beta\left(y_{R}-1\right)\right] \ln (1-2 \beta)\right]^{-1}
\end{aligned}
$$

where $\beta=G M / R c^{2}$ is the compactness parameter. The parameter $y_{R}$ is determined by solving the relevant differential equation of $y(r)$ simultaneously with the TolmanOppenheimer-Volkoff equations [40].

Moving on to the binary neutron star systems, one of the binary parameters that is well constrained by the gravitational wave detectors is the chirp mass $\mathcal{M}_{c}$, which is a combination of the component masses $[15,17]$

$$
\mathcal{M}_{c}=\frac{\left(m_{1} m_{2}\right)^{3 / 5}}{\left(m_{1}+m_{2}\right)^{1 / 5}}=m_{1} \frac{q^{3 / 5}}{(1+q)^{1 / 5}}
$$

where $m_{1}$ is the mass of the heavier component star and $m_{2}$ is the lighter's one. Hence, the binary mass ratio $q=$ $m_{2} / m_{1}$ is within $0 \leq q \leq 1$.

The information regarding the tidal effects of the system is transfered via the gravitational waves. This information is characterized by the effective tidal deformability $[15,17]$

$$
\tilde{\Lambda}=\frac{16}{13} \frac{(12 q+1) \Lambda_{1}+(12+q) q^{4} \Lambda_{2}}{(1+q)^{5}},
$$

where the key quantity $q$ characterizes the mass asymmetry. Moreover, $\Lambda_{i}$ is the dimensionless deformability defined as $[15,17]$

$$
\Lambda_{i}=\frac{2}{3} k_{2}\left(\frac{R_{i} c^{2}}{M_{i} G}\right)^{5} \equiv \frac{2}{3} k_{2} \beta_{i}^{-5}, \quad i=1,2 .
$$

Now, by replacing in Eq. (10) the value of $k_{2}$ from Eq. (7), we found that $\Lambda_{i}$ depends both on the compactness of the star as well as on the value of $y(R)$. 


\section{Results and Discussion}

In our study we used two scenarios for the value of speed of sound, the lower bound of $\left(v_{s} / c\right)^{2}=1 / 3$ and the upper one of $\left(v_{s} / c\right)^{2}=1$, and four transition densities $n_{\mathrm{tr}}=\{1,1.5,2,3\} n_{0}$ [28]. In Fig. 1 the corresponding mass-radius diagram is displayed. The red colored lines correspond to the $\left(v_{s} / c\right)^{2}=1 / 3$ limit, while the blue ones correspond to the $\left(v_{s} / c\right)^{2}=1$ limit. One can observe that each taken value of transition density leads to bifurcations in M-R diagram. Between the same kind of linestyle, the upper (lower) bound $v_{s}=c\left(v_{c}=c / \sqrt{3}\right)$ of speed of sound corresponds to higher (lower) masses. As shown in Fig. 1, the higher the transition density, the softer the EoS, with the lower limit of $\left(v_{s} / c\right)^{2}=1 / 3$ leading to a more soft EoS, compare to the $\left(v_{s} / c\right)^{2}=1$ branch. In addition, the estimation of the GW170817 event and the NICER's data are also displayed $[16,51]$. We notice that the specific gravitational-wave's origin (GW170817) information is more suitable for our study due to the fact that while the NICER's contour estimation covers almost all the cases, the neutron stars merger that was detected (GW170817), restricts the cases, excluding at least those with transition density $n_{\mathrm{tr}}=n_{0}$, for both cases of speed of sound.

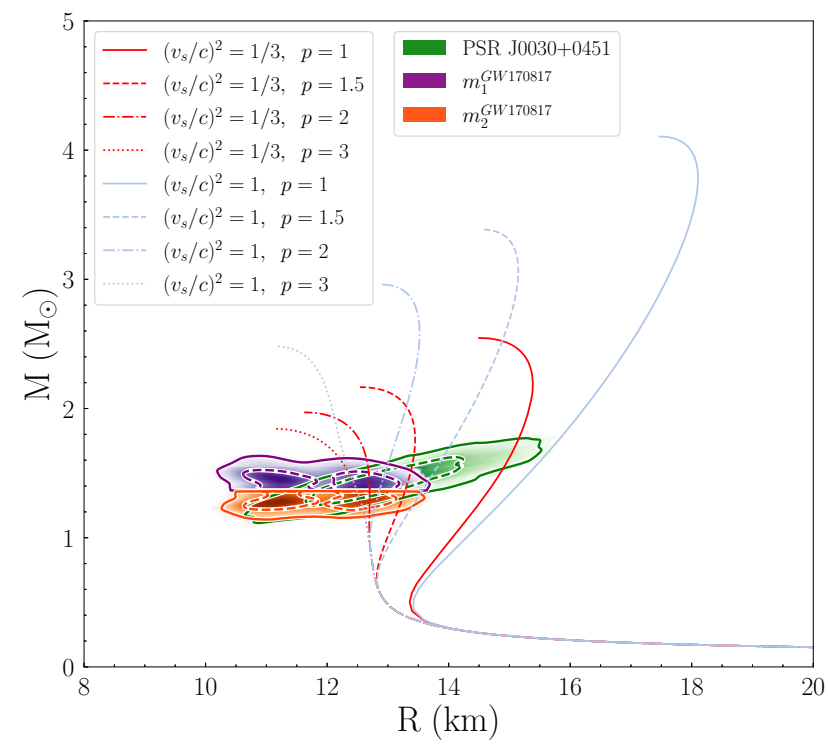

Figure 1: Mass vs radius for an isolated neutron star, and for the two cases of speed of sound bounds. The blue (red) lines correspond to the upper (lower) bound. The green diagonal shaded region corresponds to NICER's observation (data taken from Ref. [51]), while the purple upper (orange lower) shaded region corresponds to the higher (smaller) component of GW170817 event (data taken from Ref. [16]). The solid (dashed) contour lines describe the $90 \%(50 \%)$ confidence interval.

Our study takes into consideration the information from the gravitational wave emission of binary neutron stars coalescences. Hence, we used the constraints on the effective tidal deformability $\tilde{\Lambda}$, provided by the events GW170817 and GW190425 [16-18]. The chirp masses for the two events are $\mathcal{M}_{c}=1.186 M_{\odot}[15]$
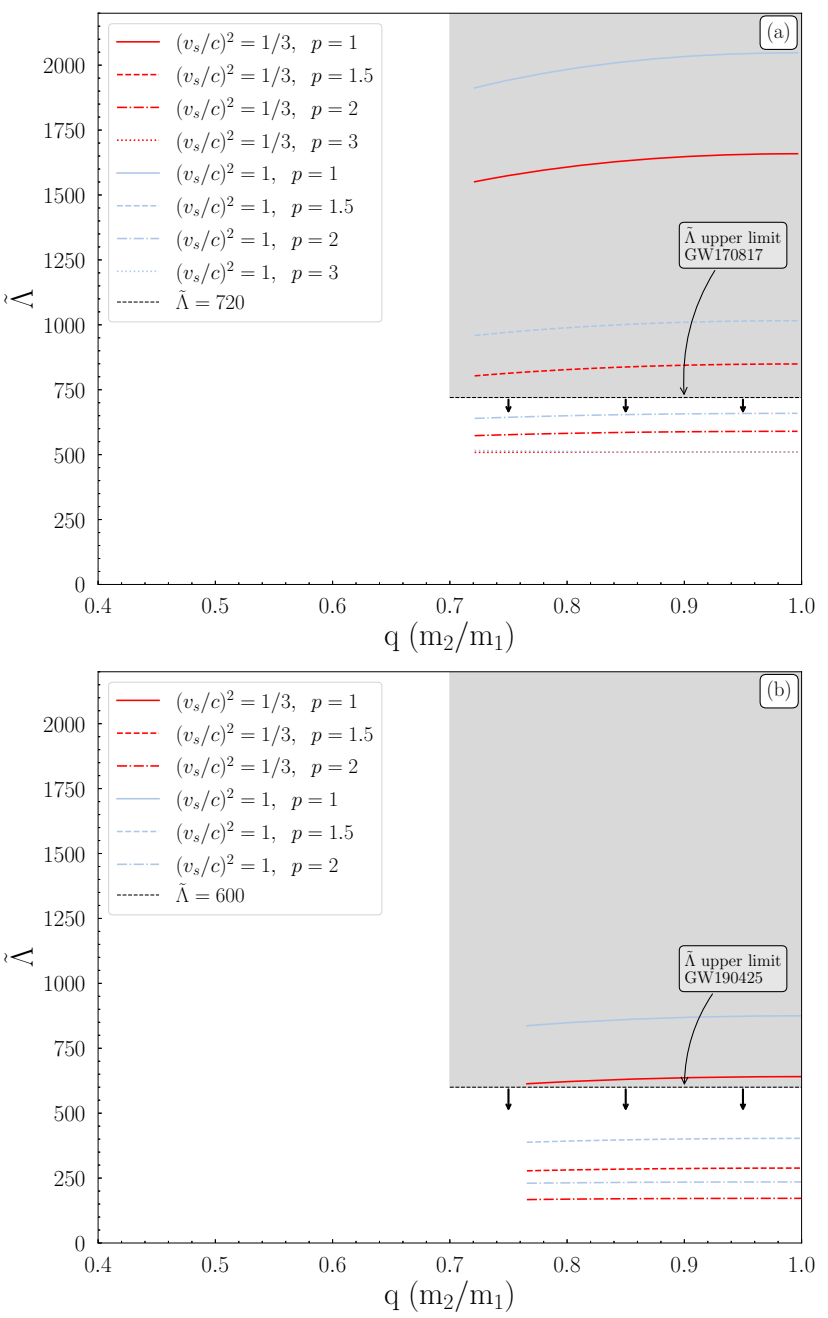

Figure 2: The tidal deformability $\tilde{\Lambda}$ as a function of the binary mass ratio $q$ for the event (a) GW170817 and (b) GW190425. The corresponding upper observation limits for $\tilde{\Lambda}$ are also indicated, with the grey shaded region marking the excluded area. The red (blue) lines correspond to the $\left(v_{s} / c\right)^{2}=1 / 3\left(\left(v_{s} / c\right)^{2}=1\right)$ limit.

and $\mathcal{M}_{c}=1.44 M_{\odot}$ [18], respectively. The component masses vary in the ranges $m_{1} \in(1.36,1.60) M_{\odot}$ and $m_{2} \in(1.16,1.36) \quad M_{\odot}$ [17] $(\mathrm{GW} 170817)$ and $m_{1} \in(1.654,1.894) \quad M_{\odot}$ and $m_{2} \in(1.45,1.654) \quad M_{\odot}$ (GW190425). We notice that we modified the range of the component masses (especially in the second event) to have an equal-mass boundary, hence $q \leq 1$.

In Fig. 2 we display the effective tidal deformability $\tilde{\Lambda}$ as a function of $q$ for both events. The upper limit on $\tilde{\Lambda}$ in Fig. 2(a), provided by GW170817, leads to the exclusion of both cases of speed of sound for transition densities $n_{\mathrm{tr}}=1,1.5 n_{0}$. By comparing to Fig. 1 , the constraints on the upper limit of $\tilde{\Lambda}$ in Fig. 2 distinguish in a more efficient way the cases that must be excluded. Furthermore, for the second event in Fig. 2(b), we observed that in general all EoSs are shifted downwards. This behavior is because of the higher value of chirp mass and the component masses. Contrary to the GW170817 event, the upper limit on $\tilde{\Lambda}$, provided by GW190425 event, ex- 

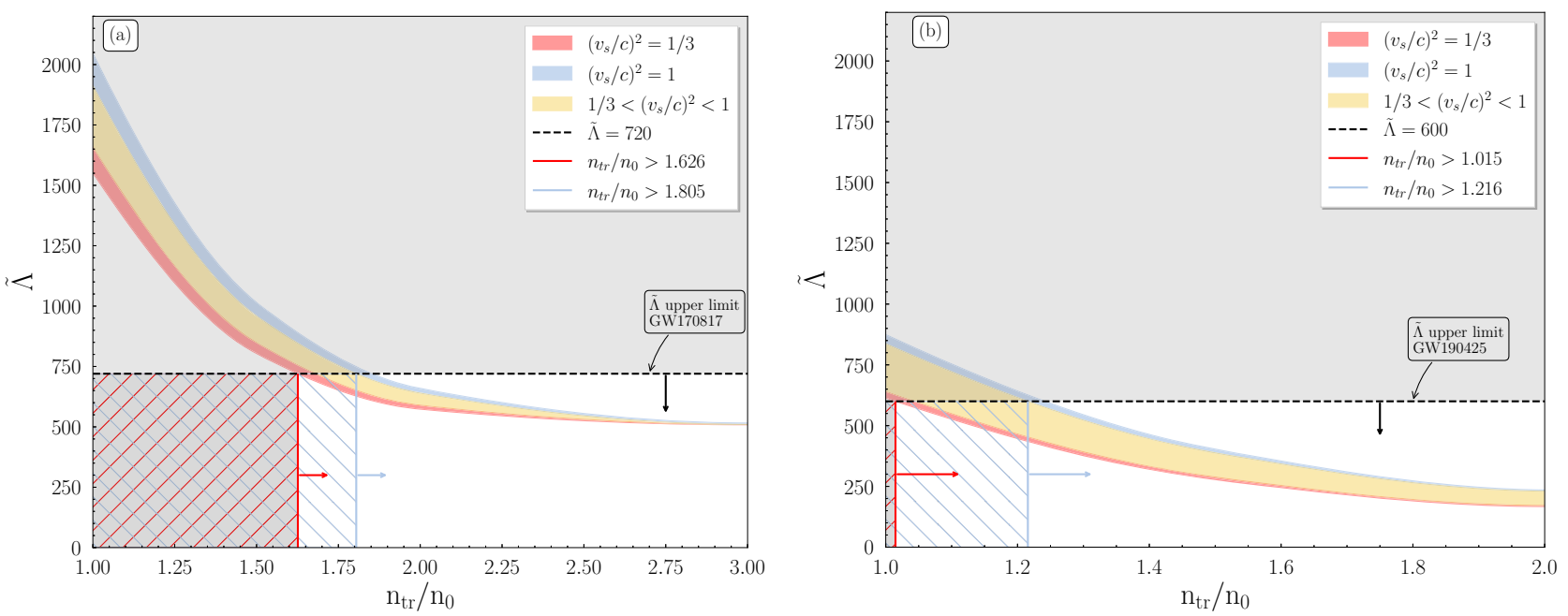

Figure 3: $\tilde{\Lambda}$ as a function of the transition density $n_{\text {tr }}$ (in units of saturation density $n_{0}$ ) at the maximum mass configuration for the two speed of sound bounds $v_{s}=c / \sqrt{3}$ and $v_{s}=c$ and for the event (a) GW170817 (left panel) and (b) GW190425 (right panel). The corresponding upper observation limits for $\tilde{\Lambda}[17,18]$ as well as the compatible lower transition density values are also indicated for both events. The red (blue) arrow marks the accepted region of transition density for the $v_{s}=c / \sqrt{3}\left(v_{s}=c\right)$ case. The red lower (blue upper) curved shaded region corresponds to the $v_{s}=c / \sqrt{3}\left(v_{s}=c\right)$ limit. The intermediate region between the two cases of speed of sound bounds is displayed with yellow color.
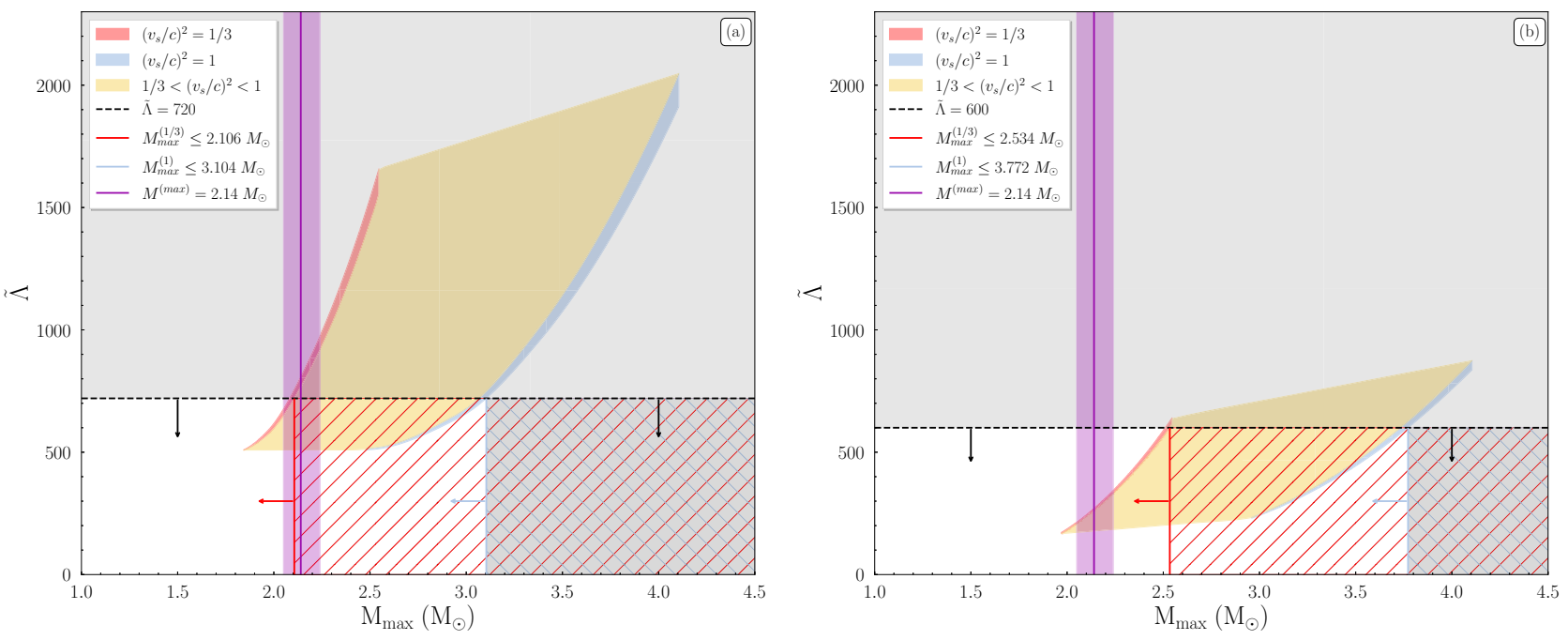

Figure 4: $\tilde{\Lambda}$ as a function of the maximum mass for the two speed of sound bounds $v_{s}=c / \sqrt{3}$ and $v_{s}=c$ and for the event (a) GW170817 (left panel) and (b) GW190425 (right panel). The corresponding upper observation limits for $\tilde{\Lambda}$ (black dashed lines with arrows, see Refs. [17, 18]), the compatible maximum mass shaded regions, for $v_{s}=c / \sqrt{3}$ (left red) case, $v_{s}=c$ case (right blue) and the middle cases (yellow), as well the current observed maximum neutron star mass $M=2.14_{-0.09}^{+0.10} M_{\odot}$ (purple shaded vertical region, see Ref. [21]) are also indicated. The red left (blue right) arrow marks the accepted region of maximum mass $M_{\max }$ for $v_{s}=c / \sqrt{3}\left(v_{s}=c\right)$ case.

cludes only the EoS with transistion density $n_{\mathrm{tr}}=n_{0}$, for both speed of sound cases. In general for both events, the EoSs with higher values of transition density $n_{\text {tr }}$ have smaller values of $\tilde{\Lambda}$. Therefore, the constraints on $\tilde{\Lambda}$ derived from gravitational-waves events favor softer EoSs. At this point we have to underline that for the GW190425 event, we removed from our study the cases with transition density $n_{\mathrm{tr}}=3 n_{0}$ because of the fact that the EoS with $\left(v_{s} / c\right)^{2}=1 / 3$ and $n_{\mathrm{tr}}=3 n_{0}$ can not reproduce the masses of this event.

In Fig. 3 we display the dependence of the effective tidal deformability $\tilde{\Lambda}$ on the transition density $n_{\text {tr }}$ at the maximum mass configuration for the two speed of sound bounds, $v_{s}=c / \sqrt{3}$ and $v_{s}=c$, and the two events GW170817 (Fig. 3(a)) and GW190425 (Fig. 3(b)). The corresponding upper observational limits for $\tilde{\Lambda}$, as well as the compatible lower transition density values, are also 
indicated in each case. The middle region corresponds to bounds on the speed of sound between the two limit cases that we studied. In the case of the GW170817 event the lower limit for the transition density is $1.626 n_{0}$ for $v_{s}=c / \sqrt{3}$ and $1.805 n_{0}$ for $v_{s}=c$. Correspondingly, for the second event GW190425 the limits are $1.015 n_{0}$ for $v_{s}=c / \sqrt{3}$ and $1.216 n_{0}$ for $v_{s}=c$. Therefore, the first event imposes more stringent constraints on the EoS. Especially, the result for the case of $v_{s}=c / \sqrt{3}$ is in agreement with other studies (see Ref. [52]). For the second event GW190425, the higher masses that characterize it lead to smaller values on $\tilde{\Lambda}$, hence the upper limit on $\tilde{\Lambda}$ cannot provide any further constraints. In Ref. [24] there is a more detailed description of the results.

In Fig. 4 we display the effective tidal deformability $\tilde{\Lambda}$ as a function of the maximum mass for the two speed of sound bounds and for both events. The corresponding upper observational limit for $\tilde{\Lambda}$ (black dashed horizontal line), the compatible maximum mass in each case (horizontal arrows), as well as the current observed maximum neutron star mass $M=2.14_{-0.09}^{+0.10} M_{\odot}$ [21] (shaded purple vertical region) are also indicated. For the first event the upper bound of $\tilde{\Lambda}$ is compatible with a maximum mass value $2.106 M_{\odot}$ for $v_{s}=c / \sqrt{3}$ and $3.104 M_{\odot}$ for $v_{s}=c$. The upper limit on $M_{\max }$ for the case of $\left(v_{s} / c\right)^{2}=1 / 3$ lays roughly inside the estimation of the observed maximum mass. There are two different points of view that antagonize one another. To be more specific, the constraints derived by the upper limit on $\tilde{\Lambda}$ lead to more soft EoSs, contrary to the observational estimations of the maximum mass of neutron stars which lead to more stiff EoSs. This difference becomes less contradictory as the speed of sound takes larger values, with the causal scenario of $v_{s}=c$ leading to a very wide area for the maximum mass. Moving on to the second event, the correspondig value for the maximum mass is $M_{\max } \leq 2.534 M_{\odot}$ for $v_{s}=c / \sqrt{3}$ and $M_{\max } \leq 3.772 M_{\odot}$ for $v_{s}=c$. As one can observe, the second event (which is in general less informative than the first one) cannot provide any further constraints.

\section{Concluding remarks}

In this work we studied possible constraints on the speed of sound (which affects the stiffness of the EoS) and transition density $n_{\mathrm{tr}}$ which are based on the recent observations of the events GW170817 and GW190425. The method which was implemented involved mainly the upper limits of the effective tidal deformability $\tilde{\Lambda}$ (estimated from the mentioned events), combined with measurements and estimations of the maximum neutron star mass. As basis of our study, we used the APR1-MDI EoS, for two boundary cases of speed of sound [24]; the lower bound of $v_{s}=c / \sqrt{3}$ and the upper one of $v_{s}=c$.

Firstly, we examined the behavior of EoSs for each case, by using the information on the component masses of GW170817 event in combination with the recent NICER's data. The M-R diagram was able to impose some robust constraints, by excluding the more stiff EoSs with $n_{\mathrm{tr}}=n_{0}$. Moreover, the upper limit on $\tilde{\Lambda}$, from $\tilde{\Lambda}-q$ diagram for both events, imposed more stringent constraints on the speed of sound bounds. Especially, the constraints from the first event (GW170817), which is more informative than the second one (GW190425), led to the additionally exclusion of EoSs with $n_{\mathrm{tr}}=1.5 n_{0}$.

Subsequently, we studied the $\tilde{\Lambda}_{\min , \max }^{(1 / 3,1)}$ as a function of $n_{\mathrm{tr}}$, where the parenthesis (min,max) denotes the minimum and maximum case respectively, for both speed of sound bounds (indicated as $(1,1 / 3)$ ). Therefore we were able to impose more stringent constraints, by using the upper limit on $\tilde{\Lambda}$ (derived from gravitational-wave observations) and taking advantage of the variety on $\tilde{\Lambda}$ for each EoS. From the first event (GW170817) we found that the speed of sound must be lower than the value $v_{s}=c / \sqrt{3}$ at least up to densities $n_{\mathrm{tr}} \approx 1.6 n_{0}$ and lower than $v_{s}=c$ up to densities $n_{\mathrm{tr}} \approx 1.8 n_{0}$. The respective values derived from the GW190425 event are $n_{\mathrm{tr}} \approx n_{0}$ for the lower speed of sound bound and $n_{\mathrm{tr}} \approx 1.2 n_{0}$ for the upper one. We notice that the constraints from the event GW170817 are more stringent compared to the GW190425 event.

Moreover, we extended our approach by treating the effective tidal deformability $\tilde{\Lambda}$ as a function of the maximum mass $M_{\max }$ for both cases of speed of sound. From the first event (GW170817) we obtained that the maximum mass should be $M_{\max } \leq 2.106 M_{\odot}$ for the $v_{s}=c / \sqrt{3}$ bound and $M_{\max } \leq 3.104 M_{\odot}$ for the upper bound $v_{s}=c$. The limit of $M_{\max } \approx 2.11 M_{\odot}$ corresponds to a transition density equal to $n_{\mathrm{tr}} \approx 1.5 n_{0}$. According to this finding, the limit $v_{s}=c / \sqrt{3}$ is in contradiction with the observational evidence on the $M_{\max }$ of neutron stars and must be violated in order to be able to simultaneously describe small values of the effective tidal deformability and high values for neutron star mass. This contradiction lays into the antagonized points of view; the upper limit on $\tilde{\Lambda}$ favors softer EoSs leading to higher values of $n_{\mathrm{tr}}$, while the observational information regarding to the maximum mass $M_{\max }$ of a neutron star requires stiffer EoSs, leading to smaller values of $n_{\mathrm{tr}}$. We notice that the second event GW190425, was not able to offer further constraints.

We postulate that future observations may offer even more rigorous constraints on the bound of the speed of sound. To be more specific, the detection of future events could provide further information on the upper limit of $\tilde{\Lambda}$, leading to more stringent constraints on $n_{\mathrm{tr}}$ and the sound speed bounds. According to our approach, the more informative events, for the lower limit of $n_{\text {tr }}$, would be those with lighter masses. Moreover, further detection of neutron stars mergers will assist both on the neutron stars maximum mass determination and its link to the speed of sound, sheding more light on the open problem of the speed of sound of dense nuclear matter and EoS.

\section{References}

[1] S. L. Shapiro and S. A. Teukolsky, Black Holes, White Dwarfs, and Neutron Stars (John Wiley and Sons, New York, 1983)

[2] N. K. Glendenning, Compact Stars: Nuclear Physics, Particle Physics, and General Relativity (Springer, Berlin, 2000) 
[3] P. Haensel, A. Y. Potekhin, and D. G. Yakovlev, Neutron Stars 1: Equation of State and Structure (SpringerVerlag, New York, 2007)

[4] S. Weinberg, Gravitational and Cosmology: Principle and Applications of the General Theory of Relativity (Wiley, New York, 1972) 47-52

[5] Ya. B. Zel'dovich and I. D. Novikov, Stars and Relativity (Dover Publications, INC, Mineapolis New York, 1971)

[6] Ya. B. Zel'dovich, Sov. Phys. JETP 14, 1143 (1962)

[7] J. B. Hartle, Phys. Rep. 46, 201 (1978)

[8] P. Bedaque and A.W. Steiner, Phys. Rev. Lett. 114, 031103 (2015)

[9] B. Reed and C.J. Horowitz, Phys. Rev. C 101 (4), 045803 (2020)

[10] C. C. Moustakidis, T. Gaitanos, C. Margaritis, and G. A. Lalazissis, Phys. Rev. C 95, 045801 (2017)

[11] E. D. Van Oeveren and J. L. Friedman, Phys. Rev. D 95, 083014 (2017)

[12] Y.-L. Ma and M. Rho, Phys. Rev. D 100, 114003 (2019)

[13] G. Baym et al., Rept. Prog. Phys. 81 (5), 056902 (2018)

[14] E. Annala, T. Gorda, A. Kurkela, J. Nattila, and A. Vuorinen, Nat. Phys. 16, 907 (2020)

[15] B.P. Abbott et al., Phys. Rev. Lett. 119, 161101 (2017)

[16] B. P. Abbott et al., Phys. Rev. Lett. 121, 161101 (2018)

[17] B. P. Abbott et al., Phys. Rev. X 9, 011001 (2019)

[18] B. P. Abbott et al., Astrophys. J. Lett. 892, L3 (2020)

[19] Z. Arzoumanian et al., Astrophys. J. Suppl. Ser. 235, 37 (2018)

[20] J. Antoniadis et al., Science 340, 1233232 (2013)

[21] H. Cromartie et al., Nat. Astron. 4, 72 (2020)

[22] M. Linares, T. Shahbaz, and J. Casares, Astrophys. J. 859, 54 (2018)

[23] P.S. Koliogiannis and C.C. Moustakidis, Phys. Rev. C 101, 015805 (2020)

[24] A. Kanakis-Pegios, P. S. Koliogiannis, and C. C. Moustakidis, Phys. Rev. C 102, 055801 (2020)

[25] R. Abbott et al., Astrophys. J. 896, L44 (2020).

[26] H. Tan, J. Noronha-Hostler, and N. Yunes, (2020) [arXiv:2006.16296 [astro-ph.HE]].

[27] A. Kanakis-Pegios, P. S. Koliogiannis, and C. C. Moustakidis, Symmetry 13, 183 (2021)

[28] Ch. Margaritis, P. S. Koliogiannis, and Ch. C. Moustakidis, Phys. Rev. D 101, 043023 (2020)
[29] C. E. Rhoades and R. Ruffini, Phys. Rev. Lett. 32, 324 (1974)

[30] V. Kalogera and G. Baym, Astrophys. J. 470, L61 (1996)

[31] S. Koranda, N. Stergioulas, and J. L. Friedman, Astrophys. J. 488, 799 (1997)

[32] N. Chamel, P. Haensel, J. L. Zdunik, and A. F. Fantina, Int. J. Mod. Phys. E 22, 1330018 (2013)

[33] C. Breu and L. Rezzolla, Mon. Not. R. Astron. Soc. 459, 646 (2016)

[34] J. Alsing, O. H. Silva, and E. Berti, Mon. Not. R. Astron. Soc. 478, 1377 (2018)

[35] D. M. Podkowka, R. F. P. Mendes, and E. Poisson, Phys. Rev. D 98, 064057 (2018)

[36] C. Xia, Z. Zhu, X. Zhou, and A. Li, (2019) [arXiv: 1906.00826 [nucl-th]]

[37] R. Feynman, N. Metropolis, and E. Teller, Phys. Rev. 75, 1561 (1949)

[38] G. Baym, C. Pethik, and P. Sutherland, Astrophys. J. 170, 299 (1971)

[39] A. Akmal, V.R. Pandharipande, and D.G. Ravenhall, Phys. Rev. C 58, 1804 (1998)

[40] S. Postnikov, M. Prakash, and J. M. Lattimer, Phys. Rev. D 82, 024016 (2010)

[41] I. Tews, J. Carlson, S. Gandolfi, and S. Reddy, Astrophys. J. 860, 149 (2018)

[42] L. Baiotti, Prog. Part. Nucl. Phys. 109, 103714 (2019)

[43] E.E. Flanagan and T. Hinderer, Phys. Rev. D 77, 021502(R) (2008)

[44] T. Hinderer, Astrophys. J. 677, 1216 (2008)

[45] T. Damour and A. Nagar, Phys. Rev. D 80, 084035 (2009)

[46] T. Hinderer, B. D. Lackey, R. N. Lang, and J. S. Read, Phys.Rev. D 81, 123016 (2010)

[47] F. J. Fattoyev, J. Carvajal, W. G. Newton, and B. A. Li, Phys. Rev. C 87, 015806 (2013)

[48] B. D. Lackey and L. Wade, Phys. Rev. D 91, 043002 (2015)

[49] J. Takátsy and P. Kovács, Phys. Rev. D 102 (2), 028501 (2020)

[50] Kip S. Thorne, Phys. Rev. D 58, 124031 (1998)

[51] M. C. Miller et al., Astrophys. J. Lett. 887, L24 (2019)

[52] S.K. Greif, G. Raaijmakers, K. Hebeler, A. Schwenk, and A.L. Watts, Mon. Not. Roy. Astron. Soc. 485 (4), 5363 (2019) 